\title{
Detecting early myocardial involvement in systemic sclerosis using cardiac magnetic resonance T1 mapping and speckle tracking echocardiography in correlation with plasma concentration of C-terminal pro-endothelin-1
}

\author{
Franck Thuny ${ }^{1}$, Claire Carmona ${ }^{2}$, Daniel Lovrić ${ }^{2 *}$, Frédéric Schnell ${ }^{2}$, Leila Potton ${ }^{2}$, Cyrille \\ Bergerot', Martine Barthelet², Sophie Thivolet², Cécile Lacote-Roiron', Laurent Boyer ${ }^{3}$, \\ Vincent Cottin ${ }^{4}$, Jean-François Cordier ${ }^{4}$, Arnaud Hot ${ }^{5}$, Jacques Ninet ${ }^{5}$, Laura Ernande ${ }^{2}$, \\ Hélène Thibault' ${ }^{2}$ Pierre Croisille ${ }^{6}$, Geneviève Derumeaux ${ }^{2}$ \\ ${ }^{1}$ Hospices Civils de Lyon, Université Claude Bernard Lyon, Lyon, France \\ ${ }^{2}$ Explorations Fonctionnelles Cardio-vasculaires, Louis Pradel Hospital, Lyon, France \\ 'INSERM U955 and Département de Physiologie, Hôpital Henri Mondor, AP-HP, Créteil, France; \\ Université Paris-Est Creteil (UPEC) \\ ${ }^{4}$ Centre de référence des maladies pulmonaires rares; Centre de compétence de l'hypertension \\ artérielle pulmonaire, Louis Pradel Hospital, Lyon, France \\ ${ }^{5}$ Department of Internal Medicine, Edouard Herriot Hospital, Lyon, France \\ ${ }^{6}$ Creatis-LRMN, UMR CNRS 5220, INSERM U630, Lyon France
}

Background: Systemic sclerosis (Ssc) leads to progressive myocardial fibrosis and subsequent alteration in function. Cardiac magnetic resonance (CMR) T1-mapping and speckle tracking echocardiography (STE) are two recent modalities able to quantify diffuse fibrosis and deformation, respectively. We aimed to determine whether these methods could detect left ventricular (LV) dysfunction at an early stage of SSc, and whether there was a relationship between myocardial alteration and plasma levels of C-terminal pro-endothelin-1 (CT-proET-1).

Methods: 54 consecutive SSc patients with normal conventional echocardiography and no CMR late gadolinium enhancement, and 16 healthy controls underwent CMR T1mapping and STE. CT-proET-1 was measured in 35 patients.

Results: As compared with the controls, SSc patients had shorter global (354 \pm 23 ms vs. $367 \pm 23, P=0.04)$ and basal inferoseptal LV post-contrast T1 values ( $336 \pm 22 \mathrm{~ms}$ vs. 353 $\pm 24, P=0.01$ ). In addition, basal inferoseptal segment in SSc patients showed decreased longitudinal peak systolic strain as compared with controls $(-16.3 \pm 4 \%$ vs $-19.6 \pm 4 \%$, $\mathrm{P}=0.009)$. $\mathrm{T} 1$ value correlated with parameters of longitudinal function $(P<0.001)$. Finally, CT-proET-1 level was higher in patients with shorter T1 value $(78 \pm 8 \mathrm{vs} .52 \pm 3 \mathrm{pmol} / \mathrm{L}$,
$P=0.03)$ and correlated with peak early diastolic strain rate $(r=0.51, P=0.002)$ within the basal inferoseptal segment.

Conclusion: CMR T1-mapping and STE can detect early abnormalities in the course of SSc, which might reflect the increase in diffuse interstitial fibrosis, and their consequences on the myocardial function.

KEYWORDS: cardiac magnetic resonance, T1-mapping, speckle tracking echocardiography, systemic sclerosis, myocardial fibrosis.

Received: $20^{\text {th }}$ Mar 2013

*Address for correspondence: Louis Pradel Hospital - Hospices Civils de Lyon, Université Claude Bernard Lyon, 28 avenue Doyen Lepine, 69677 Bron, France.

Phone: $+385-91-44-88350$

Fax: $+33-47-23-76-910$

E-mail: daniel@dlovric.net

\section{Literature}

1. Steen VD, Medsger TA Jr. Severe organ involvement in systemic sclerosis with diffuse scleroderma. Arthritis Rheum. 2000;43:2437-44.

2. Mewton N, Liu CY, Croisille P, et al. Assessment of myocardial fibrosis with cardiovascular magnetic resonance. J Am Coll Cardiol 2011;57:891-903.

3. Messroghli DR, Greiser A, Frohlich M, et al. Optimization and validation of a fully—integrated pulse sequence for modified look—locker inversion— recovery (MOLLI) T1 mapping of the heart. J Magn Reson Imaging. 2007;26:1081-6. 


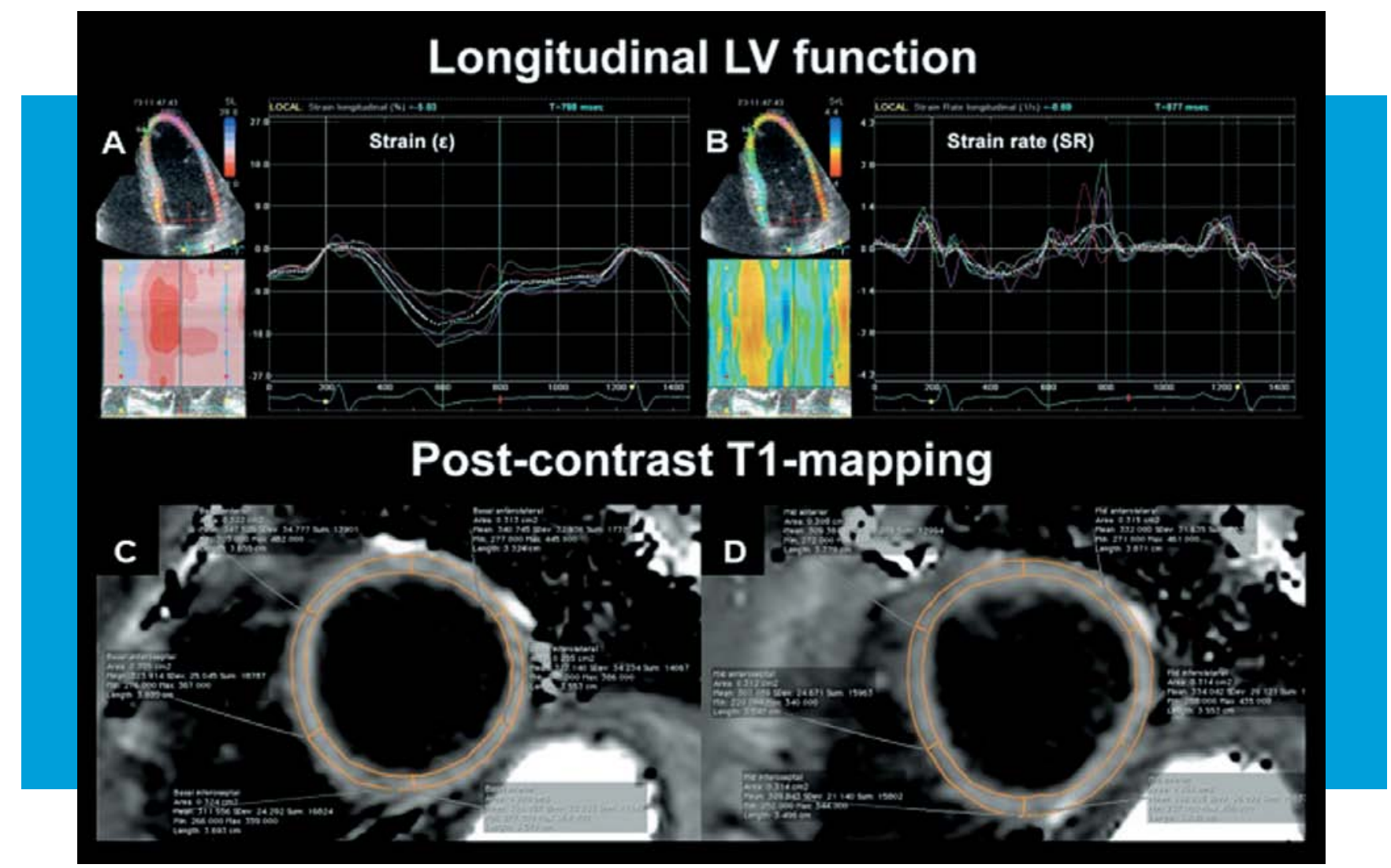

Figure 1. Examples of speckle tracking echocardiography and CMR T1-mapping measurements. LV longitudinal function: the figure shows an example of deformation imaging by speckle tracking echocardiography in the 4-chamber view. The analysis of longitudinal strain $(\boldsymbol{A})$ and strain rate $(\boldsymbol{B})$ was performed. Post-contrast T1-mapping: the figure shows the parametric $T 1$ relaxation time pixel maps of the basal $(\boldsymbol{C})$ and mid $(\boldsymbol{D})$ LV slices generated from the MOLLI sequence. The epicardial and endocardial LV contours were manually traced and each slice was divided into standard six segments, then T1 relaxation time for each segment was calculated.

4. Iles L, Pfluger H, Phrommintikul A, et al. Evaluation of diffuse myocardial fibrosis in heart failure with cardiac magnetic resonance contrast-enhanced T1 mapping. J Am Coll Cardiol. 2008;52:1574-80

5. Kepez A, Akdogan A, Sade LE, et al. Detection of subclinical cardiac involvement in systemic sclerosis by echocardiographic strain imaging. Echocardiography. 2008;25:191-7. 6. Spethmann S, Dreger H, Schattke S, et al. Two-dimensional speckle tracking of the left ventricle in patients with systemic sclerosis for an early detection of myocardial involvement. Eur Heart J Cardiovasc Imaging. 2012;13(10):863-70.

7. Leask A. The role of endothelin-1 signaling in the fibrosis observed in systemic sclerosis. Pharmacol Res. 2011;63(6):502-3. 